\title{
TRIVIAL KNOTS WITH ARBITRARY PROJECTION
}

\author{
N. SMYTHE
}

(Received 6 June 1966)

\section{Introduction}

Let $k$ be a polygonal knot in Euclidean 3 -space, $p$ a projection onto a plane. If $p \mid k$ is $1: 1$ except at a finite number of points, which are not vertices of $k$ and at which $p \mid k$ is $2: 1$, then $p(k)$ is said to be a regular projection of $k$; this means that $p(k)$ is a closed curve with a finite number of double points ("crossings") which are not points of tangency. Clearly for every polygonal knot there is a plane onto which it can be projected regularly. At each crossing of $p(k)$, the knot $k$ assigns an overcrossing arc and an undercrossing arc of the projection; conversely, if at each crossing we say which arc is an overcrossing, then there is a knot, uniquely determined up to homeomorphism, with this regular projection with the assigned overcrossings.

A knot which is the boundary of a disc is said to be trivial. It is wellknown (see for instance Alexander [1] p. 299) that given any regular projection $p(k)$ of a polygonal knot, there is a trivial knot with the same regular projection. This may readily be visualized by the method of "laying down a rope along the projection", i.e., orient the projection $p(k)$ and choose a starting point $P$ on the projection, then at a crossing of two $\operatorname{arcs} \alpha$ and $\beta$ of $p(k), \alpha$ is to be an undercrossing if it lies between $P$ and $\beta$ in the orientation of $p(k)$. The resultant knot is trivial.

Clearly the same theorem holds if $p(k)$ is a regular projection of a polygonal knot $k$ embedded in $S \times I$, where $S$ is a 2-sphere, $I$ is the closed unit interval $[0,1]$, and $p$ is the projection $S \times I \rightarrow S \times\{0\}$; for $p(k)$ is contractible in the complement of a point of $S$, so Alexander's theorem applies.

Our aim is to generalize the result to all surfaces $S$, that is, to prove

THEOREM 1. Let $S$ be a surface, orientable or not, compact or not. Let $k$ be a polygonal knot contained in, and contractible in, the interior of $S \times I$, with regular projection $p(k)$ in $S \times\{0\}$. Then there exists a knot $k^{\prime} C S \times\left[\frac{1}{4}, \frac{1}{2}\right]$ which has the same regular projection, and which bounds a disc in $S \times\left[\frac{1}{4}, \frac{1}{2}\right]$. (Since every knot with projection $p(k)$ is freely homotopic to $k$, the condition that $k$ be contractible is necessary.) 
Note that the method of "laying down rope" does not work in general; in the figure, starting at $P$ leads to the knot shown, which is not trivial in the space $S \times I$, where $S$ is an annulus.

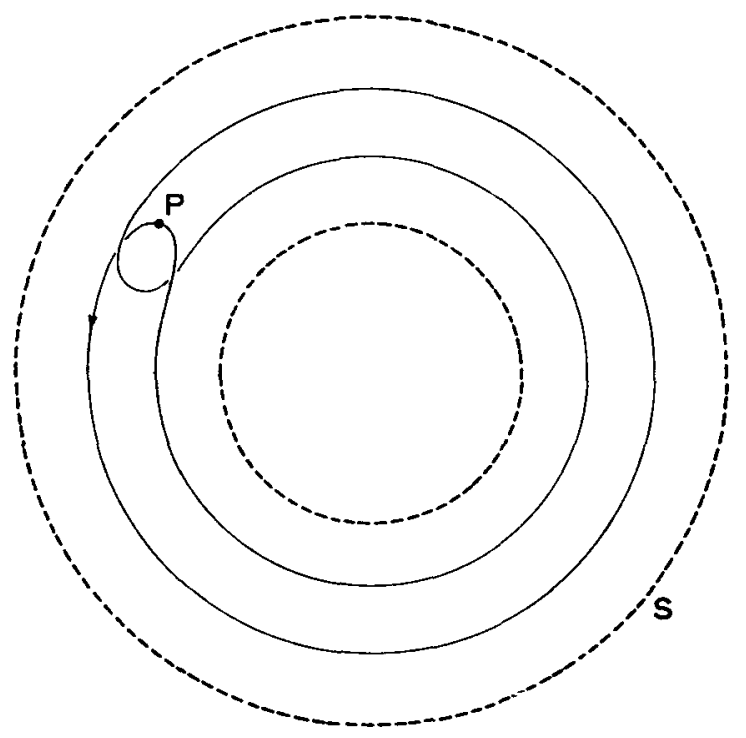

Figure 1

Unexpectedly, the proof of Theorem 1 involves the notion of a total ordering of the fundamental group $\pi(S)$ which is invariant under left multiplication. Theorem 4 states that such an ordering exists for every surface other than the projective plane. Since in fact no such ordering exists for the projective plane, a separate argument is required to prove Theorem 1 in this case; this is achieved by showing that every contractible regular projection $p(k)$ in the projective plane is contractible in the complement of some point, reducing the problem to that of the Möbius band.

We begin with a study of left invariant orderings of groups.

Definition. A total ordering of a group $G$ is said to be left invariant if whenever $u<v$ we have $a u<a v$, for all $a \in G$.

Any free abelian group may be given a left invariant ordering by choosing an arbitrary ordering of its generators, then ordering the elements of the group lexicographically.

THEOREM 2. The free group of finite or countable rank has a left invariant ordering (in fact, an ordering which is both left and right invariant). (This result is well-known, see for instance Everett and Ulam [2].) 
Proof. Any countable free group can be embedded in the free group $F$ of rank 2, so it suffices to order $F$. Let $F_{n}$ denote the $n$-th group of the lower central series of $F$, where $F=F_{1}$. Then $F_{n} / F_{n+1}$ is free abelian of finite rank, and $\bigcap F_{n}=1$.

Let $\Phi_{n}: F_{n} \rightarrow F_{n} / F_{n+1}$ be the canonical map, and let $<_{n}$ be any left invariant total ordering of $F_{n} / F_{n+1}$.

For any two distinct elements $u, v$ of $F$ there is a unique integer $n(u, v)$ such that $v^{-1} u \in F_{n(u, v)}-F_{n(u, v)+1}$. Note that $n(u, v)=n(v, u)$. Define $u<v$ if $\Phi_{n}\left(v^{-1} u\right)<_{n} 1$.

Since $<_{n}$ is a left ordering, $u<v$ and $v<u$ cannot both occur.

Transitivity of $<$ is shown as follows. If $u<v<w$, put $n=n(u, v)$ and $m=n(v, w)$. There are three cases:

(a) if $n<m$, then $w^{-1} u=\left(w^{-1} v\right)\left(v^{-1} u\right) \in F_{n}-F_{n+1}$,

and $\Phi_{n}\left(w^{-1} u\right)=\Phi_{n}\left(v^{-1} u\right)<_{n} 1$, so $u<w$;

(b) if $n>m$, then $w^{-1} u \in F_{m}-F_{m+1}$,

and $\Phi_{m}\left(w^{-1} u\right)=\Phi_{m}\left(w^{-1} v\right)<_{m} 1$, so $u<w$;

(c) if $n=m$, then $w^{-1} u \in F_{n}$,

and $\Phi_{n}\left(w^{-1} u\right)=\Phi_{n}\left(w^{-1} v\right) . \Phi_{n}\left(v^{-1} u\right)<_{n} 1$, so $\Phi_{n}\left(w^{-1} u\right) \neq 1$, i.e.

$w^{-1} u \in F_{n}-F_{n+1}$ and $u<w$.

Thus $<$ is a total ordering of the elements of $F$.

Now if $u<v$ and $a \in F$, then $(a v)^{-1}(a u)=v^{-1} u$, so $a u<a v$; thus we have a left invariant ordering. In fact, because we chose the subgroups $F_{n}$ to be members of the lower central series, we also have $\Phi_{n}\left(a^{-1} v^{-1} u a\right)=$ $\Phi_{n}\left(v^{-1} u\right)$, so $u a<v a$; thus the ordering is also a right ordering.

Q.e.d.

COROLLARY. The fundamental group of any open surface or surface with boundary has a left invariant ordering.

For the fundamental group of such a surface is free.

The proof of the following result is now clear.

THEOREM 3. Let $N$ be a normal subgroup of the group $G$. If both $N$ and $G / N$ have left invariant orderings, then so does $G$.

For we may combine these orderings as in the previous theorem:

if $w^{-1} u \in N$, define $u<w$ if $w^{-1} u<1$ in the ordering of $N$;

if $w^{-1} u \in G-N$, define $u<w$ if the image of $w^{-1} u<1$ in $G / N$.

This defines a left ordering of $G$; it should be noted that this ordering is not usually right invariant.

We apply this result to find a left invariant ordering of the groups of surfaces. 
THEOREM 4. Let $G$ be the fundamental group of any surface other than the projective plane. Then $G$ has a left invariant total ordering.

Proof. The closed surfaces are the only outstanding cases. The orientable surfaces have fundamental group of the form

$$
\left(a_{1}, b_{1}, \cdots, a_{h}, b_{h}: \prod a_{i} b_{i} a_{i}^{-1} b_{i}^{-1}=1\right)
$$

while the non-orientable surfaces have fundamental group of the form

$$
\left(a_{1}, \cdots, a_{k}: a_{1} a_{1} a_{2} a_{2} \cdots a_{k} a_{k}=1\right)
$$

for integral $h \geqq 0$ and $k \geqq 1$.

If $h=0$ (the surface is the 2 -sphere), the group is trivial, so has a left invariant total ordering; if $k=1$, the surface is the projective plane, and the group is cyclic of order 2, so has no left invariant total ordering.

For $h \geqq 1$ and $k \geqq 2$, there is a homomorphism of the group onto the infinite cyclic group. The kernel of this homomorphism is the fundamental group of a covering space of the surface with infinitely many sheets; this covering space is therefore an open surface, and the kernel is a free group. The preceding theorem gives the result.

In connection with this result, I am informed by G. Baumslag that the groups of the orientable closed surfaces can be given an ordering which is both left and right invariant (for they are residually free, so can be embedded in a direct product of free groups). On the other hand, this is not true of the groups of non-orientable surfaces; it is easily seen that the group of the Klein bottle $(a, b: a a b b=1)$ has no 2 -sided ordering.

We proceed to the proof of Theorem 1.

The cases $S=$ sphere and $S=$ plane are covered by Alexander's theorem. We shall defer the case $S=$ projective plane to the next section.

For any other surface $S$, let $M$ be the interior of $S$, and denote by $\tilde{M} \cong R \times R \times I$ the universal covering space of $M \times I$, where $R$ denotes the real line. Let $e: \tilde{M} \rightarrow M \times I$ be the covering map.

$p(k)$ is covered by closed curves $k_{u}=T_{u}\left(k_{1}\right)$, where $k_{1}$ is the particular such curve through the base point of $\tilde{M}$ and $T_{u}$ is the covering translation corresponding to the element $u \in \pi(M \times I) \approx \pi(S)$; we assume $\pi(S)$ has been given a left invariant ordering.

Let $\tilde{p}: R \times R \times I \rightarrow R \times R \times\{0\}$ be the projection over $p$. The collection of closed curves $\left\{k_{u}\right\}$ has only double points as singularities (which are not points of tangency); we shall construct simple closed curves $\left\{\tilde{k}_{u}\right\}$ in the interior of $\tilde{M}$ which project regularly onto $\left\{k_{u}\right\}$ under $\tilde{p}$ and such that $\tilde{k}_{u}=T_{u}\left(\tilde{k}_{1}\right)$. 
Choose an orientation for $p(k)$, thus inducing an orientation in each $k_{u}$. Choose disjoint neighbourhoods of the double points of the collection $k_{u}$, so small that in each neighbourhood there are two simple arcs of the collection with one crossing; at a crossing $P$ of $k_{1}$ with itself we denote these arcs by $A(P)$ and $B(P)$, where $A(P)$ is the arc preceding $B(P)$ in the orientation of $k_{1}$ starting at the base point of $\tilde{M}$; at a crossing $P$ of $k_{1}$ and $k_{u}$, we denote the arcs by $C(P)$ and $D(P)$, where $C(P)$ is the arc in $k_{1}$ and $D(P)$ is the arc in $k_{u}$.

a) If $(r, s, 0)$ is a point of $k_{1}$ not in any of the chosen neighbourhoods, or is a point of an $\operatorname{arc} A(P)$, or is a point of an arc $C(P)$ where $P$ is a double point of $k_{1}$ and $k_{u}$ with $u>1$, then we choose $\left(r, s, \frac{1}{4}\right)$ to be a point of $\tilde{k}_{1}$.

b) If $(r, S, 0)$ is a point of an arc $B(P)$, or is a point of $C(P)$ where $P$ is a double point of $k_{1}$ and $k_{u}$ with $u<1$, then as the point of $\tilde{k}_{1}$ lying over $(r, s, 0)$ we choose a point $\left(r, s, \frac{1}{4}+t\right), 0<t \leqq \frac{1}{4}, t$ varying continuously so as to be $\frac{1}{4}$ at $P$ and approaching 0 as $(r, s, 0)$ approaches the boundary of the neighbourhood of $P$.

The result is a simple closed curve $\tilde{k}_{1}$ with regular projection $\tilde{p}\left(\tilde{k}_{1}\right)=k_{1}$. Let $\tilde{k}_{u}=T_{u}\left(\tilde{k}_{1}\right)$.

These are disjoint simple closed curves. For suppose $Q \in \tilde{k}_{u} \cap \tilde{k}_{v}$. Then $T_{u^{-1}}(Q)=P \in \tilde{k}_{1} \cap \tilde{k}_{u^{-1} v}$ and $T_{v^{-1}}(Q)=P^{\prime} \in \tilde{k}_{v^{-1} u} \cap \tilde{k}_{1}$. We may assume $u>v$. Since $\tilde{p}(P)$ is a double point of $k_{1}$ and $k_{u^{-1} v}$ with $u^{-1} v<1$, we have by construction that $P \in R \times R \times\left\{\frac{1}{2}\right\}$; on the other hand, $\tilde{p}\left(P^{\prime}\right)$ is a double point of $k_{1}$ and $k_{v^{-1}}$ with $v^{-1} u>1$, so by construction $P^{\prime} \in R \times R \times\left\{\frac{1}{4}\right\}$. But $T_{u} P=T_{v} P^{\prime}$, and covering translations leave the third co-ordinate invariant, giving a contradiction.

Next we notice that if $\tilde{k}_{u}$ lies above $\tilde{k}_{v}$ (in the sense of the third coordinate) at any crossing of $k_{u}$ and $k_{v}$, then it does so at all their crossings, for this depends only on the fact that $u>v$. $\tilde{k}_{w}$.

Furthermore if $\tilde{k}_{u}$ lies above $\tilde{k}_{v}$ and $\tilde{k}_{v}$ lies above $\tilde{k}_{w}$ then $\tilde{k}_{u}$ lies above

Thus we may depress the curves $\tilde{k}_{u}$ for $u<1$, and lift the curves $\tilde{k}_{v}$ for $v>1$ without disturbing $\tilde{k}_{1}$; that is, precisely, there is an isotopy $h_{t}: M \rightarrow \tilde{M}$, with $h_{0}$ being the identity and such that $h_{1}$ has the following properties:

i) $h_{1}\left(\tilde{k}_{u}\right) \subset R \times R \times\left(0, \frac{1}{4}\right)$ for $u<1$

ii) $h_{1}\left(\tilde{k}_{1}\right)=k_{1}$ remains fixed in $R \times R \times\left[\frac{1}{4}, \frac{1}{2}\right]$

iii) $h_{1}\left(\tilde{k}_{v}\right) \subset R \times R \times\left(\frac{1}{2}, 1\right)$ for $v>1$

iv) $p h_{1}\left(\tilde{k}_{w}\right)=k_{w}$, and is a regular projection, for all $w$.

Then $h_{1}\left(\tilde{k}_{1}\right)$ has been isolated in the 3-cell $R \times R \times\left[\frac{1}{4}, \frac{1}{2}\right]$. The lifting process described in a) and b) above is exactly the process of "laying down a rope" when restricted to the crossings of $k_{1}$ with itself; by Alexander, $\tilde{k}_{1}$ 
is therefore contractible in the complement of $\bigcup_{u \neq 1} \tilde{k}_{u}$, bounding a nonsingular disc $D$ in $R \times R \times\left[\frac{1}{4}, \frac{1}{2}\right]$ not meeting any other $\tilde{k}_{u}$.

Since $\tilde{k}_{1} \cap T_{u}\left(\tilde{k}_{1}\right)=\emptyset, e\left(\tilde{k}_{1}\right)$ is a simple closed curve in $M \times I$ with regular projection $p e\left(\tilde{k}_{1}\right)=p(k) . e\left(\tilde{k}_{1}\right)$ bounds the singular disc $e(D)$. However since $e(D) \cap T_{u}\left(\tilde{k}_{1}\right)=\emptyset, e(D)$ has at most internal singularities. By Dehn's Lemma [3], $e(D)$ may be modified in a neighbourhood of its singularities (and therefore within $M \times\left[\frac{1}{4}, \frac{1}{2}\right]$ ) so that $e\left(\tilde{k}_{1}\right)$ bounds a nonsingular disc.

This completes the proof of Theorem 1 except for the case $S=$ projective plane.

We now assume $p(k)$ is the regular projection onto $S \times\{0\}$ of a polygonal knot in $S \times I$, where $S$ is the projective plane. The following Lemma reduces this case to that of the Möbius band, thus completing Theorem 1.

Lemma. If $p(k)$ is contractible in the projective plane, then it is contractible in the complement of some point.

Proof. From the closed curve $p(k)$ we form a finite number of disjoint simple closed curves $C_{1}, \cdots, C_{m}$, the "Seifert circles" of $p(k)$, as follows.

At each crossing $P$ of $p(k)$ choose a neighbourhood $N(P)$ in the projective plane which is homeomorphic to a disc and is so small that it contains no other crossings; $p(k) \cap N(P)$ consists of two arcs $\alpha$ and $\beta$ crossing at $P$. Choose a point $A$ on $\alpha$ preceding $P$ in the orientation of $p(k)$, and a point $B$ on $\beta$ succeeding $P$ in the orientation of $p(k)$. Join $A$ to $B$ by an arc $\gamma$ in $N(P)$ not meeting $p(k)$ in any other points, and delete the open $\operatorname{arcs} A P$, $P B$.

Diagrammatically:

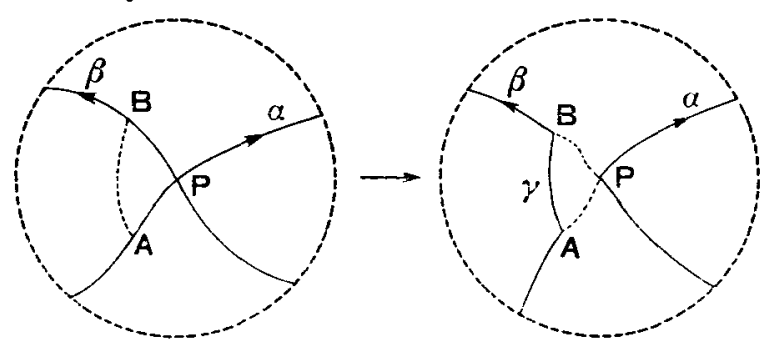

Figure 2

After this is done at each crossing, we have constructed a number of closed curves which have no crossings, and whose union differs from $p(k)$ only within a small neighbourhood of the crossings of $p(k)$; these are the desired curves $C_{1}, \cdots, C_{m}$. 
It is easily seen that $p(k)$ is homotopic within the set $p(k) \cup \cup N(P)$ to a product of conjugates of $C_{1}, \cdots, C_{m}$.

Considered as 1-cycles of $S, \sum C_{i}$ is homologous to $p(k)$, which is contractible in $S$; thus $\sum C_{i} \sim 0$. Now $H_{1}(S)$ is cyclic of order two, so that an even number of the cycles $C_{i}$ are non-bounding; suppose $C_{1}$ and $C_{2}$ are nonbounding. $S-C_{1}$ is an open disc so $C_{2} \subset S-C_{1}$ is contractible, contradicting our assumption. Thus every $C_{i}$ is null-homologous and therefore contractible in $S$.

$C_{i}$ separates $S$ into two connected regions with $C_{i}$ as common boundary; by the usual Euler characteristic argument, one of these pieces is a Möbius band, the other is a disc $D_{i}$. It may happen that $D_{i}$ contains a Seifert circle $C_{j}$; since $D_{i}$ contains no Möbius band, we must have $D_{j} \subset D_{i}$. Let $D_{1}, \cdots, D_{n}$ be the "outermost" discs, i.e. those not contained in any other disc of the collection; these discs are pairwise disjoint.

To the subspace $\bigcup_{i=1}^{n} D_{i}$ we add the discs $N(P)$, for each crossing $P$ of $p(k)$, to get a connected proper subspace $S^{\prime}$ of $S$ containing $p(k)$. Since $p(k)$ is homotopic within $S^{\prime}$ to a product of conjugates of the $C_{i}^{\prime}$ 's, and each $C_{i}$ is contractible within $S^{\prime}$, we have that $p(k)$ is contractible in $S^{\prime}$.

Thus $p(k)$ is contractible in the complement of a point of $S$, i.e., within a Möbius band contained in $S$.

It should be noted that the analogous result is not true for other closed surfaces. Thus if $S$ is any closed surface whose universal covering space is the Euclidean plane, let $\tilde{k}$ be a simple closed curve in the plane enclosing a sheet over $S$; then $k$, the image of $\tilde{k}$ in $S$, is a contractible closed curve of $S$ which is not contractible in the complement of any point. Clearly $\tilde{k}$ may be chosen so that $k$ is a regular projection of a polygonal knot in $S \times I$.

\section{Applications}

The Alexander theorem is the basis for the definition of the Gordian invariant, or unknotting number, of a knot in 3-space. For every regular projection of a polygonal knot, some number of crossings may be changed to give a trivial knot; the Gordian invariant is the smallest such number over all projections. This definition may now be extended to knots projected onto surfaces other than the plane.

As an application in another direction, we have

CoRollary. Let $T$ be a torus, and $k_{1}$ and $k_{2}$ be oriented disjoint knots in the interior of $T \times I$ such that the projection $p\left(k_{1} \cup k_{2}\right)$ on $T \times\{0\}$ is a regular projection of the link $k_{1} \cup k_{2}$ with $p\left(k_{1}\right) \cap p\left(k_{2}\right) \neq \emptyset$. Then if $k_{1}$ is homotopic to $k_{2}$, there exist knots $k_{1}^{\prime}, k_{2}^{\prime}$ in the interior of $T \times I$ with oriented regular projections $p\left(k_{1}^{\prime}\right)=p\left(k_{1}\right), p\left(k_{2}^{\prime}\right)=p\left(k_{2}\right)$, such that $k_{1}^{\prime} \cup k_{2}^{\prime}$ bounds a non- 
singular polyhedral annulus in the interior of $T \times I$ (and therefore there is an isotopy of $T \times I$ taking $k_{1}^{\prime}$ onto $k_{2}^{\prime}$ which leaves the boundary of $T \times I$ fixed).

Proof. Let $P$ be a point of $p\left(k_{1}\right) \cap p\left(k_{2}\right)$. Choose a small neighbourhood of $P$ in $T \times\{0\}$ containing no other crossings, meeting $p\left(k_{1}\right)$ in an arc $\alpha$, and $p\left(k_{2}\right)$ in an arc $\beta$. As in fig. 3 , choose two parallel arcs $\phi$ and $\psi$ joining $\alpha$ and $\beta$, cutting off arcs $\sigma$ and $\rho$ from $\alpha$ and $\beta$ respectively. Let $D$ denote the disc in $T \times\{0\}$ bounded by $\phi, \psi, \sigma$ and $\rho$. Let $k^{*}$ denote the closed curve produced from $p\left(k_{1}\right)$ and $p\left(k_{2}\right)$ by this operation, with orientation induced from $p\left(k_{1}\right)$.

Then $k^{*}$ is homologous to $k_{1}-k_{2}$ in $T \times I$, so is homologous to zero. But $H_{1}(T \times I) \approx \pi(T \times I)$, so $k^{*}$ is contractible. Also $k^{*}$ is the regular projection of some knot.

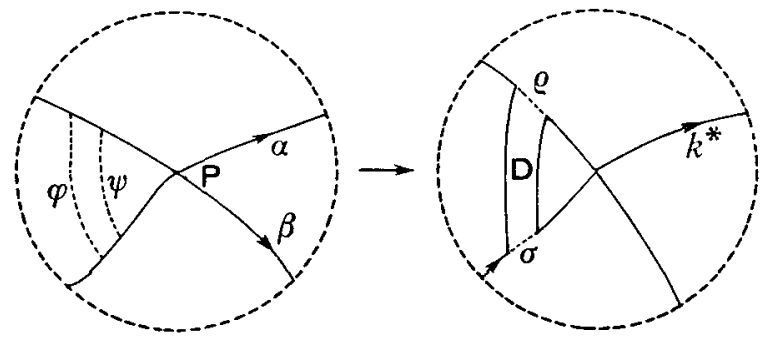

Figure 3

Then there is a knot $k^{\prime \prime}$ in $T \times\left[\frac{1}{4}, \frac{1}{2}\right]$ bounding a polyhedral disc $D^{\prime \prime}$, the points of $k^{\prime \prime}$ being of the form $\left(q, \frac{1}{4}\right)$ for $(q, 0) \in k^{*}$ except near crossings, and such that $D^{\prime \prime}$ meets $T \times\left[\frac{1}{4}, \frac{1}{2}\right]$ only at points of $k^{\prime \prime}$. In particular $k^{\prime \prime}$ contains arcs $\phi^{\prime}, \psi^{\prime}$ contained wholly in $T \times\left\{\frac{1}{4}\right\}$ and which lie over $\phi, \psi$ respectively. Let $\sigma^{\prime}, \rho^{\prime}, D^{\prime}$ in $T \times\left\{\frac{1}{4}\right\}$ be the sets lying over $\sigma, \rho, D$ respectively.

Since $D^{\prime} \cap D^{\prime \prime}=\dot{D}^{\prime} \cap \dot{D}^{\prime \prime}=\phi \cup \psi^{\prime}, D^{\prime} \cup D^{\prime \prime}$ is a non-singular polyhedral surface with two boundaries $k_{1}^{\prime}$ and $k_{2}^{\prime}$ with regular projections $p\left(k_{1}\right)$ and $p\left(k_{2}\right)$. By calculation of its Euler characteristic, this surface is an annulus

This result gives

q.e.d.

COROLlary. Let $V$ be a solid torus (Vollring) with boundary torus $T$, and let $p(k)$ be a regular projection on $T$ of some knot $k$ in a neighbourhood of $T$ homeomorphic to $T \times I$. If $k$ has winding number 1 in $V$, then there is a knot $k$ in an arbitrarily small neighbourhood of $p(k)$ which is a core of $V$.

Proof. For any given $\delta>0$, choose a neighbourhood of $T$ homeomorphic to $T \times I$ lying within $\delta$ of $T$, with projection map $p^{\prime}: T \times I \rightarrow T$ such that $p(k)$ is the regular projection $p^{\prime}\left(k^{\prime}\right)$ of some knot $k^{\prime}$ in this neighbourhood. Choose a simple closed curve $l$ on $T$ with winding number 1 in $V$ such that $l \cap p(k) \neq 0$. 
Then there exist knots $k^{*}, l^{*}$ with regular projections $p^{\prime}\left(k^{*}\right)=p(k)$, $p^{\prime}\left(l^{*}\right)=l$ respectively, and an isotopy of $T \times I$ taking $l^{*}$ onto $k^{*}$ leaving the boundary of $T \times I$ fixed. This may be extended to an isotopy of $V$ leaving $T$ fixed. Since $l$ is simple, $l^{*}$ bounds an annulus with $l$ and is therefore a core of $V$. Thus $k^{*}$ is also a core of $V$.

\section{References}

[1] J. W. Alexander, "Topological invariants of knots and links", Trans. Am. Math. Soc., 30 (1928), 275-306.

[2] C. J. Everett and S. Ulam, "Ordered Groups". Trans. Am. Math. Soc., 57 (1945), 208-216.

[3] C. D. Papakyriakopoulos, "On Dehn's lemma and the asphericity of knots". Ann. of Math., 66, (1957), 1-26.

University of New South Wales 